\title{
Marvin Müller
}

\section{Die fiktionale Markierung der Legende von Tarpeia bei Livius $(1,11,5-9)$}

\begin{abstract}
In Livy's report, in Book 1 of his annals, of the mythical traitor Tarpeia, who, according to tradition, had betrayed the Romans to their enemies the Sabines out of lust for money and was then murdered by her accomplices, it is possible to interpret both the principal figure and her motives in an open way. With reference to the praefatio, it will here first be argued that the term fabula is important in this context and that a manuscript reading rejected by most modern editions should be retained. This makes it possible to interpret the discussion of story-variants, which is presented after this term, as a narrative functional instrument that causes the reader to doubt the veracity of the tradition about the criminal Tarpeia. In conclusion two further passages from the following chapter will be considered, which, through their semantic ambiguity, are well-suited to support the ambivalent character-drawing proposed here. Understood this way, the Tarpeia episode refers, through its narrative mode, to myth's lack of historical reliability, a theme already raised in the praefatio.
\end{abstract}

Keywords: Livius, Tarpeia, fabula, Variantendiskussion, Textkritik

DOI 10.1515/phil-2014-0019

The ancient literary tradition concerning the early kings can perhaps be best likened to a contemporary Hollywood blockbuster concerning some major historical episode. Though the movie is beautifully produced and tells a story in a powerful and memorable fashion, the viewer is left wondering what parts of the film are historically accurate, and what parts are distortions or outright fabrications introduced into the plot to make the movie more appealing ${ }^{1}$.

1 Forsythe (2005) 78.

Marvin Müller: LMU München, Abt. f. Griech. und Lat. Philologie, Geschwister-Scholl-Platz 1, 80539 München, E-Mail: marvin.mueller@klassphil.uni-muenchen.de 
Der Vergleich, den der Historiker Gary Forsythe zwischen antiker Geschichtsschreibung und modernen Spielfilmen zieht, scheint besonders gut auf einen Historiographen der Antike zu passen: Titus Livius. Denn dass Livius recht freimütig mit seinen Quellen und deren historischer Authentizität umgegangen ist, gehört seit langer Zeit zum Allgemeinwissen über den Autor und war in der älteren Forschung Gegenstand von Kritik². Allerdings ist bemerkenswert, dass die Kritik der mangelnden Historizität des Erzählten bei Livius so alt ist wie die Quelle, auf die sie sich bezieht. So antizipiert der Autor selbst in der praefatio zu seinem Geschichtswerk genau diesen Vorwurf, der dann auch später immer wieder an seinen Text herangetragen worden ist ${ }^{3}$ :

Quae ante conditam condendamve urbem poeticis magis decora fabulis quam incorruptis rerum gestarum monumentis traduntur, ea nec adfirmare nec refellere in animo est ${ }^{4}$.
Was vor Gründung der Stadt oder dem Vorhaben, sie zu gründen, passender mit dichterischen Legenden als mit unverbrüchlichen Denkmälern der Taten überliefert wird, das will ich weder bekräftigen noch zurückweisen.

Begründet wird der Einsatz der poeticae fabulae, von denen hier die Rede ist, mit dem beträchtlichen Alter der Ereignisse ante urbem conditam, das eine faktentreue Wiedergabe unmöglich mache. Doch zeigt sich bald, dass die fabula als Bestandteil der historiographischen Erzählung längst nicht, wie es die Formulierung in der praefatio vermuten ließe, auf diese im Verhältnis recht kurze Zeitspanne beschränkt bleibt - im Gegenteil: In einer zweiten Vorrede, die das erste Buch der zweiten Pentade einleitet, erfährt der Leser schließlich, dass die gesamte bisherige Schilderung letztlich auf einer unsicheren Quellenlage beruht hat, weil

2 Abwertend äußert sich in dieser Beziehung noch der RE-Artikel von Klotz (1972) 831-840 („Verhältnis des L. zum Stoffe“). Aber auch in der aktuellen Forschungsdiskussion wird die ältere Kritik immer wieder aufgegriffen, allein um die „,kopernikanische Wende‘ in der Livius-Forschung“ (Pausch 2011, 8) zu illustrieren (so z.B. bei Chaplin/Kraus 2009). Pausch selbst will schließlich wieder eine vermittelnde Position zwischen der alten Tradition der Quellenforschung und neueren Ansätzen, die die Annalen primär als literarischen Text lesen, einnehmen (vgl. Pausch 2011, 8).

3 Livius' praefatio war und ist Gegenstand oder Ausgangspunkt zahlreicher Publikationen, auch hinsichtlich der Frage nach dem fiktionalen Gehalt der Annalen insgesamt (z. B. Walsh 1955). Ein close reading der praefatio bietet Moles $(22009=1993)$. Seine Deutung zu praef. $6(64-66)$ kommentiert und erweitert Miles (1995) 16-20. Einen aktuellen Überblick über Forschungsliteratur zur praefatio insgesamt bietet Moles (22009) 86f. [= Addendum der Hgg.].

4 Liv. praef. 6. - Textgrundlage bei Zitaten der ersten Pentade ist die Ausgabe von Ogilvie (1974), wobei konsonantisches $, \mathrm{u}^{\mathfrak{}} \mathrm{zu}, \mathrm{v}^{\star}$ geändert wird. Alle Übersetzungen sind, sofern nicht anders vermerkt, meine eigenen. 
wegen des Stadtbrandes 390 v. Chr. ${ }^{5}$ kaum mehr Aufzeichnungen der bisher behandelten Zeit existieren.

Quae ab condita urbe Roma ad captam eandem Romani [...] gessere, [...] quinque libris exposui, res cum vetustate nimia obscuras velut quae magno ex intervallo loci vix cernuntur, tum quod rarae per eadem tempora litterae fuere, una custodia fidelis memoriae rerum gestarum, et quod, etiam si quae in commentariis pontificum aliisque publicis privatisque erant monumentis, incensa urbe pleraeque interiere ${ }^{6}$.
Was die Römer von der Gründung Roms bis zur Einnahme desselben [...] vollbracht haben, [...] habe ich in fünf Büchern dargelegt - alles Dinge, die sowohl allzu sehr durch ihr Alter verdunkelt sind, als wenn sie aus großer Entfernung kaum erkannt werden, besonders aber auch, weil es in dieser Zeit nur vereinzelte Schriftzeugnisse gab, welche die einzig verlässliche Bewahrung der Erinnerung an die geschichtlichen Ereignisse bieten, und weil von diesen, wenn überhaupt welche in den Aufzeichnungen der Priester und anderen öffentlichen und privaten Zeugnissen existieren, die meisten beim Brand der Stadt verloren gingen.

Das Prinzip des ,Hollywood-Stils‘ in $A b$ urbe condita findet, um im eingangs zitierten Bild Forsythes zu bleiben, seine Anwendung also nicht nur, wie von Livius einleitend formuliert, bis zur Gründung der Stadt, und auch nicht, wie Forsythe beklagt, bis in die römische Königszeit, sondern bis weit in die Zeit der römischen Republik hinein.

Allerdings zeigen bereits die zitierten Passagen aus Livius' Geschichtswerk, dass der Erzähler in $A b$ urbe condita die Unzulänglichkeit der Quellen bisweilen selbst thematisiert und auf diese Weise dem Leser ihren möglicherweise fiktionalen Charakter erst bewusst macht ${ }^{7}$. Es drängt sich die Frage auf, welche Funktion die fiktionale Markierung von Erzählsequenzen im historiographischen Kontext speziell bei Livius hat. Die Kommentarliteratur beschränkt sich hierzu häufig auf folgende Aussage: Livius unterscheide die Fakten von dem, was er für Legende hielt ${ }^{8}$. Im Folgenden möchte ich dafür argumentieren, dass diese zweifellos

5 Dieses Jahr setzt Livius für die Katastrophe an der Allia an (statt 386 v. Chr.). - Vgl. Hillen (1991) 512.

6 6, 1, 1-2. - Text: OCT (1970), edd. C. F. Walters/R. S. Conway.

7 Eine nützliche Übersicht über Formulierungen, mit denen dies geschieht, findet sich bei Steele (1904). - Eine Auswahl: unpersönliche Ausdrücke wie vix credibile est (19f.) oder Ausdrücke wie ferunt, dicitur o.ä. (21f.), die Termini fabula und fama (23f.) und varianteneinleitende Wendungen wie quidam [narrant], sunt qui o.ä. (26f.).

8 Als Illustration gebe ich einige Beispiele für Erläuterungen zum Begriff fabula aus unterschiedlichen Livius-Kommentaren: Ogilvie (1965) 675: ,fabula for L. means a story to which he attaches little belief“; Ross (1997) 89: ,fabula: the very choice of the word suggests that Livy gives the tale scant belief.“; Weissenborn/Müller ( $\left.{ }^{8} 1965\right)$ 179: „fabula...] das Folgende betrachtet Livius als 
naheliegende Antwort nicht immer der Bedeutung gerecht werden kann, die Fiktionsmarkierungen auch für die Leserlenkung haben können.

Um dies zu begründen, werde ich anhand einer Episode aus $A b$ urbe condita beispielhaft $\mathrm{zu}$ zeigen versuchen, wie durch solche Markierungen Verunsicherung über die Deutungsmöglichkeiten eines historischen Ereignisses erzeugt wird und wie diese Verunsicherung schließlich auch $\mathrm{zu}$ einer Reflexion über den möglicherweise fiktionalen Charakter des Berichteten anregen kann.

II

In dieser Episode aus dem ersten Buch der Annalen, die sich um 700 v. Chr. zugetragen haben soll, geht es um den Verrat der Römerin Tarpeia an ihrem eigenen Volk, der dazu führt, dass die feindlichen Sabiner das römische Kapitol einnehmen können.

Novissimum ab Sabinis bellum ortum multoque id maximum fuit; nihil enim per iram aut cupiditatem actum est, nec ostenderunt bellum prius quam intulerunt. Consilio etiam additus dolus. Sp. Tarpeius Romanae praeerat arci. Huius filiam virginem auro corrumpit Tatius ut armatos in arcem accipiat; aquam forte ea tum sacris extra moenia petitum ierat. Accepti obrutam armis necavere, seu ut vi capta potius arx videretur seu prodendi exempli causa ne quid usquam fidum proditori esset. Additur fabula ${ }^{9}$, quod volgo Sabini aureas armillas magni ponderis brachio laevo gemmatosque magna specie anulos habuerint, pepigisse eam quod in sinistris manibus haberent; eo scuta illi pro aureis donis congesta. Sunt qui eam ex pacto tradendi quod in sinistris manibus esset derecto arma petisse dicant et fraude visam agere sua ipsam peremptam mercede ${ }^{10}$.
Zuletzt begannen die Sabiner den Krieg und dieser war mit Abstand der größte. Denn nichts wurde von ihnen aus Zorn oder Gier ausgeführt und sie gaben auch keine Anzeichen für den Krieg, ehe sie ihn eröffneten. Diesem Vorsatz wurde noch eine List hinzugefügt. Sp. Tarpeius war Vorsteher der römischen Feste. Dessen Tochter, eine junge Frau, bestach nun Tatius mit Gold, damit sie die Sabiner bewaffnet in die Feste einließ. Sie war zu diesem Zeitpunkt zufällig außerhalb der Stadtmauern unterwegs gewesen, um Wasser für heilige Handlungen zu beschaffen. Die so empfangenen Soldaten töteten die Frau, indem sie sie mit Waffen überschütteten, entweder weil die Feste lieber als gewaltsam genommen erscheinen sollte oder um ein Exempel zu statuieren, dass einem Verräter niemals Vertrauen geschenkt würde. Dem wird eine Geschichte hinzugefügt: Dass sie

Sage“; Heurgon (1963) 56: „T.-L., ayant rapporté brièvement cette tradition déplaisante, mais qu’il tient pour historique, la distingue de la légende (fabula)“; Levene (1993) 184: „About this story, however, Livy expresses great scepticism: he refers to it as a 'fabula'“.

9 fabula plerique edd.: fabulae (-le) codd. - Zur ausführlichen Diskussion dieser Stelle s. Abschnitt IV.

$101,11,5-9$. 
sich, weil die Sabiner im Allgemeinen goldene Armbänder von großem Gewicht am linken Arm und mit Edelsteinen geschmückte Ringe von großer Schönheit hatten, als Lohn ausbedungen habe, was sie in ihren linken Händen hielten. Und da seien auf sie anstelle von goldenen Geschenken die Schilde gehäuft worden. Weiterhin gibt es welche, die behaupten, dass sie, indem sie forderte, zu übergeben, was sie in ihren linken Händen hielten, geradewegs nach den Waffen verlangt habe und dass sie, weil sie <den Angreifern> in betrügerischer Absicht $\mathrm{zu}$ handeln schien, selbst durch ihren eigenen Lohn getötet wurde.

Es ist leicht erkennbar, dass diese Textpassage eine ungewöhnlich hohe Anzahl an Fiktionsmarkierungen auf engstem Raum versammelt. Als erstes wecken die potentiellen Mordmotive, die mithilfe von seu - seu gegliedert werden, Argwohn. Dem Leser wird bewusst: Warum die junge Frau von den Sabinern getötet wurde, obwohl sie ihren Teil der Vereinbarung, nämlich den Sabinern Zutritt zur römischen Feste zu gewähren, offensichtlich eingelöst hat, kann der Erzähler nicht mit hinreichender Sicherheit beantworten, weil gleich zwei mögliche Alternativen präsentiert werden. Entweder waren die Sabiner auf ihr eigenes Ansehen bedacht und wollten vertuschen, dass sie den Angriff mit List und nicht mit Gewalt ausgeführt hatten, oder dem Mord liegt ein moralischer Impetus zugrunde: Kein Verräter verdient es, zu leben.

Doch damit sind die Varianten der Geschichte noch nicht ausgeschöpft. Denn der Diskussion um die Motive des Mordes an Tarpeia wird daraufhin eine fabula beigegeben. In dieser Version der Geschichte gibt schließlich Tarpeia den Sabinern einen Anlass, sie zu töten, indem sie deren Schmuck als Belohnung für ihren Verrat fordert. Diese dritte mögliche Erklärung für den Mord ist ebenfalls fiktional markiert, weil der Terminus der fabula, wie in der praefatio gesehen, impliziert, dass es nicht lohnt, die Historizität des Berichteten zu untersuchen (nec adfirmare nec refellere). Zusätzlich sollte der Wechsel des Genus Verbi beachtet werden: Wenn der Erzähler mit den Worten novissimum ab Sabinis bellum ortum multoque id maximum fuit eröffnet, haben wir als Leser keinen Grund daran zu zweifeln, dass der Krieg mit den Sabinern tatsächlich der größte der Kriege in diesem Jahr gewesen sei. Doch spätestens bei additur fabula und dem Wechsel ins Passiv zieht sich der Erzähler zurück. Wer fügt hier etwas hinzu? Welche Glaubwürdigkeit kann diese Stimme beanspruchen?

Mit sunt qui folgt schließlich noch eine weitere Fiktionsmarkierung, die andernorts regelmäßig dadurch, dass der Erzähler mitteilt, es gebe zu dem bisher 
vorgelegten Bericht noch eine andere Lesart, Zweifel aufkommen lässt, ob das im Hauptnarrativ Berichtete tatsächlich der Wahrheit entspricht oder ob nicht vielmehr der nun präsentierten Variante der Vorzug zu geben ist ${ }^{11}$. An dieser Stelle wirken die Umstände des Mordes nur noch nebulöser. Der Leser erfährt: Es gibt wiederum Stimmen, die noch ein anderes Mordmotiv in Anschlag bringen. In diesem Szenario ist auf einmal Tarpeia keine Verräterin, sondern eine Heldin. Mit ihrer Forderung nach dem, was die Feinde in der linken Hand hielten, habe sie nicht etwa den Schmuck, sondern die Waffen verlangt. Die Sabiner hätten aber den Betrugsversuch bemerkt und ihr die Waffen nicht ausgehändigt, sondern sie stattdessen getötet.

Vier mögliche Mordmotive und keine Lösung - die drei Fiktionsmarkierungen und die Varianten, die mit ihnen verbunden sind, führen in die Aporie. Ein älterer Kommentar vermerkt seinerzeit dazu noch lapidar: „Livy’s naïve selection among the stories is somewhat amusing. " ${ }^{12}$ Für die ungewöhnliche Häufung von Varianten möchte ich aber, insbesondere im Horizont der bereits erwähnten „kopernikanischen Wende“13 der Forschung, eine andere Deutung anbieten.

\section{III}

Um zunächst einschätzen zu können, welcher Status den jeweiligen Varianten des Tarpeia-Mordes in der Überlieferung beizumessen ist, sollte vorab versucht werden zu klären, womit die römische Leserschaft den Namen Tarpeia verbunden haben könnte. Die Quellen vermitteln $\mathrm{zu}$ dieser Frage ein eindeutiges Bild: Tarpeia galt als „Erzverräterin“14, deren Geschichte wohl schon bei Q. Fabius Pictor Eingang in die Historiographie gefunden hat ${ }^{15}$. Tatsächlich darf sie damit auch als erste namhafte Verbrecherin des jungen römischen Reiches gelten. Auch Properz stellt sie - wenige Jahre nach Livius ${ }^{16}$ - ins Zentrum einer seiner Elegien, auch hier im Übrigen als Verräterin, jedoch begeht sie hier den Verrat (dem Genre der Elegie gemäß) aus Liebe, nämlich zum Sabiner Tatius ${ }^{17}$. Wichtiger noch als die literarische Tradition dieser Figur ist aber das Nachleben ihres Namens in der römischen Topographie: An der Stelle des Verrats auf dem Kapitol war nämlich

11 Vgl.z.B. 5, 41, 3.

12 Greenough (1976) 37.

13 Pausch (2011) 8.

14 Zimmermann (2002) 29.

15 Vgl. Stevenson (2011) 179.

16 Vgl. Heurgon (1963) 12. - Heurgon spricht etwas ungenau von „à la même époque“.

17 Prop. 4, 4. - Zu weiteren literarischen Verarbeitungen der Figur vgl. Kowalewski (2002) $32 f$. 
ein steiler Abhang nach ihr rupes Tarpeia (oder Tarpeium saxum) benannt ${ }^{18}$, eine Klippe, von der aus Verbrecher in die Tiefe gestürzt wurden. Es spricht daher viel dafür, dass Tarpeia und die alte Geschichte ihres Verrats nicht nur Teil des kulturellen Gedächtnisses Roms gewesen sind, sondern durch die aitiologische Herleitung der rupes Tarpeia zumindest der römischen Stadtbevölkerung stets unmittelbar präsent waren ${ }^{19}$.

Auf diese rekonstruierten historisch-kulturellen Rahmenbedingungen nimmt auch die Geschichtsschreibung Bezug: Um das römische Allgemeinwissen zu bestätigen, kann sie die Aitiologie reproduzieren ${ }^{20}$. Dies gilt insbesondere im Rahmen der Geschichte der römischen Frühzeit, da diese den Historiographen als Repertoire für Ursprungsgeschichten aller Art diente ${ }^{21}$. Nach Pausch und Poucet war auch Livius diesem aitiologischen Interesse verpflichtet; neben dem bereits erwähnten arbeiten sie zwei weitere (implizite) Aitien allein in dem kurzen Abschnitt zu Tarpeia heraus ${ }^{22}$. In der Art und Weise, wie Livius dem Leser das Aition präsentiert, lässt sich aber vermuten, dass neben der offiziellen Bekräftigung des Überlieferten auch - und dies vor allem mit Blick auf die letzte Variante, die Tarpeia zu einer Heldin macht - eine Rehabilitation Tarpeias ,durch die Hintertür in Aussicht gestellt wird.

Die Wirkung der Textstelle, so lautet die vorgeschlagene These, ist also doppelt: Zunächst wird die konventionelle Version von der Verräterin Tarpeia mit verschiedenen Varianten referiert, ganz gemäß der antizipierten Erwartungshaltung. Auf diese Weise erscheint uns der Erzähler als glaubwürdig, denn er zeigt, dass er einerseits zahlreiche unterschiedliche Quellen rezipiert hat, und erweist sich damit als Experte, und dass er andererseits an der traditionellen Überlieferung festhält. Andererseits wächst der Zweifel am Wahrheitsgehalt der Erzählung mit jeder Fiktionsmarkierung. Die einzige Variante, die der Überlieferungstradition gänzlich zuwider läuft, ist nun augenscheinlich die letzte, die sich als einzige dadurch auszeichnet, dass Tarpeia keine Verbrecherin ist. Gerade dieser Variante kommt innerhalb der Erzählsequenz eine besondere Bedeutung zu.

18 Vgl. hierzu Richardson (1992) s.vv. Tarpeia Rupes, Tarpeius Mons 377f.

19 Vgl. Pausch (2008) 54 Fn. 81 und Stevenson (2011) 179.

20 Feichtinger nennt diese aitiologischen Geschichten „soziale, ethisch normgebende PseudoMythen“, die „auf keinen Fall weggelassen werden durften“ (Feichtinger 1992, 16, orig. teilw. kursiv).

21 Vgl. Pausch (2008), bes. 41f.

22 Nämlich zum einen die Besiedlung des Kapitols durch die Sabiner (vgl. Pausch 2008, 53f.), zum anderen die Herkunft des römischen Rechtskonzepts der bona fides (vgl. Poucet 1992, 295). 


\section{IV}

Um diese These begründen zu können, werde ich zunächst eine textkritische Frage diskutieren, die für die Beurteilung der Variantendiskussion an dieser Stelle maßgeblich ist. Konkret geht es um das Wort fabula $(1,10,8)$, eine kleine, aber bedeutsame Textverbesserung des Humanisten Glarean (16 Jh.). Alle Kodizes überliefern statt fabula den Dativ fabulae ${ }^{23}$. Der Textkorrektur Glareans wird aber in allen modernen Ausgaben der Vorzug gegeben ${ }^{24}$. Glarean, der auch die Syntax der folgenden Sätze für korrupt hält, begründet seinen Eingriff in den überlieferten Text mit einem inhaltlichen Argument. Er hält es für unwahrscheinlich, dass die ganze Erzählung von der List der Sabiner als bloße fabula verstanden werden soll:

Omnes in dandi casu legunt, fabulae, quasi quae praemiserit autor de caede Tarpeiae, fabulam videri velit. Itaque ego in nominandi casu, fabula legam, ut sequentia fabulam vocet, non praecedentia ${ }^{25}$.
Alle Textzeugen haben fabulae im Dativ, als ob der Autor wollte, dass all das, was er über den Mord an Tarpeia vorausgeschickt hat, als fabula gelten soll. Deshalb will ich lieber fabula im Nominativ lesen, sodass er das Folgende fabula nennt, nicht das Vorangegangene.

Die Auffassung Glareans scheinen die modernen Editoren zu teilen. Ogilvie begründet seine Entscheidung für den Nominativ mit dem analog konstruierten huic loco inseritur fabula $(5,21,8)^{26}$. Jedoch erscheint dieser Beleg etwas beliebig: Genauso könnte man argumentieren, dass der Halbsatz inde fabulae adiectum est (5, 22, 6), der nur wenig später folgt, den Dativ der Handschriften stütze. Die Entscheidung für die Konjektur Glareans hat beträchtliche Auswirkungen auf die Interpretation. Denn auf diese Weise wird das Hauptnarrativ deutlich von der fiktional markierten fabula abgegrenzt, die zur Variante der Überlieferung wird.

Im Kontext der vorangegangenen Überlegungen erscheint die handschriftlich überlieferte Variante allerdings nicht mehr emendationsbedürftig, wird doch die gesamte Textpassage unter Verwendung von Fiktionsmarkierungen erzählt, deren Vielzahl nur eines bedeuten kann: Die gesamte Überlieferung an dieser Stelle ist eine fabula, denn die tatsächlichen Geschehnisse um den Mord an Tarpeia können nicht rekonstruiert werden. Der Zweifel des Lesers, der bereits durch die

23 Oder fabule. - Vgl. Ogilvie (1974) 16.

24 Vgl. Ogilvie (1974) 16; Walters/Conway (1960) z. St. und Heurgon (1963) 56. - Nur Greenough (1976) 37, der eine ältere (zu seiner Zeit aktuelle) Weissenborn-Ausgabe abdruckt, hat noch fabulae, welches von den Editoren in späteren Auflagen jedoch ebenfalls zu fabula geändert wird (vgl. Weissenborn/Müller ${ }^{12} 1965,121$ ).

25 Glareans Livius-Kommentar von 1540 (Blatt 7), Onlinezugriff möglich, s. Bibliographie.

26 Vgl. Ogilvie (1974) 16. 
Fiktionsmarkierung seu - seu verunsichert war, wird nun durch additur fabulae also nur noch weiter verstärkt, nicht erst erzeugt. Daher schlage ich vor, die Konjektur Glareans, die diesen Effekt wissentlich tilgt, nicht zu berücksichtigen und stattdessen den Handschriften zu folgen.

Damit ändert sich gleichzeitig die Struktur der Textpassage, insofern nun die Fiktionsmarkierung additur fabulae keine unabhängige Variante mehr einleitet, sondern lediglich Zusatz der konventionellen Geschichte vom Verrat Tarpeias ist. Die letzte Variante der Tarpeia-Geschichte wird dadurch besonders exponiert: Sie ist nicht mehr eine beliebige Variante von vieren, sondern die einzige, die neben der konventionellen Erzählung noch Bestand hat. An die Stelle des Nebeneinander von mehreren Mordmotiven tritt also eine Synkrisis von zwei kontradiktorischen Auslegungen ein und derselben Geschichte:

\section{Gliederung der Mordmotive bei Annahme des Gliederung der Mordmotive bei Annahme des konjekturalen additur fabula handschriftlichen additur fabulae}

1a) Vertuschung des Verrats1b) warnendes exemplum

1a) Vertuschung des Verrats

1b) warnendes exemplum

2) Goldgier Tarpeias

1c) Goldgier Tarpeias

3) Enttarnung der List Tarpeias

2) Enttarnung der List Tarpeias

\section{V}

Zum textkritischen Befund passen die stilistischen Besonderheiten, die die Tarpeia-Episode auszeichnen und ebenfalls auf eine Ambivalenz in der Beschreibung der Figur deuten.

Die livianische Erzähltechnik ist vor allem in den romanhaften Episoden wiederholt mit Begriffen wie 'Dramatik' und 'Anschaulichkeit' charakterisiert wor$\operatorname{den}^{27}$. Auch die Tarpeia-Geschichte bietet, so könnte man vermuten, Stoff für eine solche dramatische Darstellung. Umso überraschender ist zunächst der Befund: Die Bearbeitung bei Livius erfüllt diese Erwartung nicht. Auf Ogilvie wirkt die Erzählung vor allem im Gegensatz zur vorausgehenden Hersilia-Episode als ,undramatically and briefly“28 und zeichnet sich durch ihre auffällige Schlichtheit aus ${ }^{29}$.

27 Zur Technik der ćvópyદı bei Livius vgl. z. B. Pausch (2011) 209-223, der in diesem Zusammenhang exemplarisch den Bacchanalienskandal $186 \mathrm{v}$. Chr. analysiert.

28 Ogilvie (1965) 74.

29 Vgl. ibid. und Heurgon (1963) 56. 
Dieser zunächst vielleicht überraschende Eindruck kann wie folgt erklärt werden: Die Raffung zahlreicher Handlungen (Tatius' günstige Gelegenheit zum Bestechungsversuch, die Umstände des eigentlichen Verrats, der Tod der Verräterin) bewirkt eine Reduktion auf wenige stichwortartige Notizen. Zwischen Bestechung und Verrat beispielsweise ist die Beschreibung der Vorbereitungen beider Seiten für den Verrat ausgelassen. Bis auf den letzten Aspekt, den Tod der Verräterin und die Umstände ihres Todes, die, wie gezeigt, Gegenstand der Variantendiskussion sind und allein dadurch eine intensivere Auseinandersetzung erfahren, ist keine dieser Handlungen $\mathrm{zu}$ einer Episode ausgebaut, die besonderes Interesse oder Spannung erzeugen könnte. Dazu passt, dass weder Tatius noch Tarpeia jemals in einer direkten Rede, die ihr Verhalten veranschaulichen könnte, das Wort ergreifen und ihre Motive erklären. Zwar wird man einwenden können, dass Livius auch sonst ,schlechten“ Charakteren kaum direkte Reden zugesteht, aber hier reicht die minimalistische Komposition sogar so weit, dass wir nicht einmal den Namen der eigentlichen Hauptfigur, Tarpeia, erfahren. Sie ist lediglich huius filia, die Tochter des Sp. Tarpeius. Allein durch diesen Hinweis wird die Identifikation der Figur überhaupt möglich. Darüber hinaus rückt das deiktische ea sie nur ein einziges Mal auch grammatisch, als Subjekt des Satzes, ins Zentrum des Geschehens. Ansonsten ist sie (auch grammatisch) das Objekt einer Handlung (huius filiam [...] corrumpit Tatius) oder befindet sich in Abhängigkeit eines übergeordneten Subjekts (sunt qui eam [...] arma petisse dicant).

Gerade die Anonymisierung der eigentlichen Hauptfigur, also Tarpeias, kann man als charakteristische Wirkung dieser gerafften Darstellungsweise verstehen. Folgerichtig entsteht durch ein solches literarisches Vorgehen auch keine Anschaulichkeit, ganz im Gegenteil. Durch die äußerste Kürze entstehen Zeitsprünge und Ellipsen, welche ihrerseits inhaltliche Leerstellen bewirken, die jeder Leser mit seiner eigenen Interpretation füllen muss, um zu einem Verständnis der Textstelle zu gelangen: Wie gelang es Tarpeia, die bewaffneten Sabiner unbemerkt in der römischen $a r x$ zu empfangen ${ }^{30}$ ? Deutet die Verwendung des Begriffes virgo darauf, dass Tarpeia eine Vestalin war, und bereitete sie deshalb heilige Handlungen vor ${ }^{31}$ ? Hinzu kommen logische Ungereimtheiten: Mit welcher Berechtigung konnte Tarpeia als Lohn für den Verrat den Schmuck der Sabiner verlangen, obwohl Tatius sie vorher bereits mit Gold bestochen hatte (auro corrumpit Tatius)? Diese und andere Fragen, die man mit einigem Recht an den Text herantragen könnte, werden nicht beantwortet. Die Hintergründe und Zusammenhänge der

30 Vgl. Kowalewski (2002) 24 Fn. 36.

31 So vermuten Ogilvie (1965) 75 und Kowalewski (2002) $23 f$. 
Geschichte werden nicht entfaltet ${ }^{32}$. Der Fokus liegt vielmehr auf jenem Teil der Erzählung, auf den die Aufmerksamkeit des Lesers auch durch die Fiktionsmarkierungen gelenkt wird: auf der Diskussion um die Frage, ob Tarpeia eine Verräterin war. Auf diese Weise trägt also auch die stilistische Komposition dazu bei, dass die Variantendiskussion, die mit sunt qui eingeleitet wird, das eigentliche Thema des Abschnittes ist. Die Zuspitzung der beiden Versionen ist allein durch die Raffung von Zeit und Handlung fokussiert, nicht weil die Verwendung erzählerischer Mittel Spannung erzeugen würde ${ }^{33}$.

Allerdings hat Ogilvie im Rahmen seines Kommentars gezeigt, wie kunstvoll die Erzählung von Tarpeia im Kontext der römischen Auseinandersetzung mit den Sabinern eingebettet ist ${ }^{34}$. Sie kann, so Ogilvie, als zweiter Akt eines internen Dramas, welches das Schicksal der geraubten sabinischen Frauen bei den Römern zum Gegenstand hat, gelesen werden ${ }^{35}$. Außerdem macht Wiseman auf die Hypothese aufmerksam, dass unterschiedlichen Episoden des ersten Buches, unter anderem auch der Darstellung der Tarpeia-Geschichte, (verlorene) fabulae praetextae zu Grunde gelegen haben sollen ${ }^{36}$.

Die fabula vom Verrat der Tarpeia kann also nicht nur als legendenhafte Erzählung gedacht werden, sondern unter Umständen sogar als etwas, was zur Darbietung auf der Bühne geeignet ist, was jedoch nicht bedeutet, dass die Geschichte tatsächlich in einer dramatischen Bearbeitung des Stoffes auf die Bühne gebracht wurde (und Livius auf diese Bearbeitung auch anspielt), sondern lediglich, dass sie das Potenzial hierfür bietet ${ }^{37}$.

Denkt man sich die von Livius in brüskem Stil referierte Geschichte als Dramenhandlung, wird die Variante in 1, 10, 9 (sunt qui...) zum alternativen Ende dieses Dramas. Hier wäre Tarpeia ein grundsätzlich moralisch integrer Charakter, weil sie die Feinde ihrem Volk ausliefern will und zu diesem Zweck nur zum

32 Kowalewski macht dafür den Patriotismus des Autors verantwortlich. - Vgl. Kowalewski (2002) 24.

33 Spannung entsteht aus diesem Grund zwar nicht in Hinblick auf den Tarpeia-Mord, wohl aber durch ihn: Dadurch, dass die Umstände ihres Todes verhältnismäßig ausführlich erörtert werden, wird der Fortgang der eigentlichen Handlung, und damit die spannende Frage retardiert, wie es den Römern gelingt, sich, obwohl die Sabiner durch Tarpeia in den römischen Rückzugsraum eindringen konnten, gegen die Feinde zu behaupten.

34 Vgl. Ogilvie (1965) $64 f$.

35 Vgl. Ogilvie (1965) 74.

36 Vgl. Wiseman (1998) 4. Diese Position wird noch von Wiseman selbst für die bereits kurz erwähnte Textstelle 5, 21, 8-9 vertreten: „[T]he Roman historian distanced himself from one particular episode in terms which clearly imply that he thought it had been invented for a play.“ (Wiseman 1998, 2). - Dagegen Keaveney (2006).

37 Hier ist Keaveney wohl Recht zu geben (vgl. Keaveney 2006, 510f.). 
Schein auf deren List eingeht. Am Schluss muss sie für diese Hybris mit dem Tod bezahlen. Dem Leser wird auf diese Weise auch aus dramaturgischer Perspektive eine Alternative zur überlieferten Geschichte der Verräterin präsentiert - und auch sie liefert einen guten Dramenplot.

\section{VI}

Tarpeia bleibt mit den beiden dichotomen Deutungsversuchen ihrer Todesumstände - war sie Verbrecherin oder Heldin? - und den damit verbundenen Fiktionsmarkierungen eine ambivalente Figur. Die Betrachtung zweier kurzer Notizen aus dem folgenden zwölften Kapitel, welche nochmals Bezug auf die Ereignisse des Tarpeia-Mordes nehmen, soll einen letzten Beleg für diese These liefern:

Sunt qui eam [...] arma petisse dicant et [...] sua ipsam peremptam mercede. Tenuere tamen arcem Sabini .... ${ }^{38}$
Weiterhin gibt es welche, die behaupten, dass sie [...] geradewegs nach den Waffen verlangt habe, und dass sie [...] selbst durch ihren eigenen Lohn getötet wurde. Jedenfalls war die Burg jetzt in der Hand der Sabiner ... ${ }^{39}$

Im ersten Beispiel übersetzt Hillen das Adverb tamen, welches die mit sunt qui eingeleitete Variante mit dem Hauptnarrativ, also der folgenden Erzählung von der Rückeroberung der römischen Feste, verbindet, mit ,jedenfalls‘. Die konzessive Grundbedeutung kommt auf diese Weise nicht zum Ausdruck ${ }^{40}$. Alternativ könnte man übersetzen: ,Dennoch konnten die Sabiner die Feste einnehmen.

Die zweite Textstelle ist Teil eines Gebets, das Romulus an Jupiter richtet und in dem er diesen um Beistand im bevorstehenden Kampf anruft:

Arcem iam scelere emptam Sabini habent ...41

Die Burg ist bereits durch Bestechung in der Hand der Sabiner ... ${ }^{42}$

$381,12,1$.

39 Hillen (1987) 37.

40 Die Kommentare stützen Hillens Übersetzung: vgl. Weissenborn/Müller (121965) 122; Ogilvie (1965) 77; Heurgon (1963) 56. Alle fassen tamen i.S.v. OLD tamen (1) auf.

$411,12,4$.

42 Hillen (1987) 37. 
Ambig ist hier das participium coniunctum $\mathrm{zu}$ arcem, scelere emptam. Da das Motiv des Bestechens mit emere anklingt ${ }^{43}$, übersetzt Hillen schlicht mit ,durch Bestechung'. Doch ist das Lateinische eigentlich viel allgemeiner: Scelus deckt die ganze Bandbreite des Verbrechens und der Freveltat ab. Welches scelus ist hier also gemeint? Der Verrat der Tarpeia oder doch nicht viel eher der Mord an ihr, mit dem die Sabiner sich die Feste ,(er)kauften'? Aus Romulus' Gebet lässt sich jedenfalls nicht eindeutig schließen, dass er Tarpeia als Verräterin ansah ${ }^{44}$.

Trotz mehrerer Indizien wird Tarpeia aber auch nicht als tragische Heldin rehabilitiert; vielmehr liegt die Entscheidung in diesem Kriminalfall beim Leser ${ }^{45}$. Die Erzählung wahrt dadurch an dieser Stelle die Balance zwischen der Bestätigung des römischen Allgemeinwissens und dessen Infragestellung und trägt damit, ähnlich wie bei einer ,if not-situation ${ }^{\times 46}$, zur kurzfristigen Verunsicherung des Lesers bei: Was wäre, wenn Tarpeia gar keine Verräterin (wie allgemein angenommen) gewesen ist, sondern in Wahrheit die Sabiner betrügen wollte?

\title{
Verzeichnis der verwendeten Literatur
}

\author{
J. D. Chaplin/C. S. Kraus, Introduction, in: Dies. (Hgg.), Livy (Oxford Readings in Classical Stu- \\ dies), Oxford 2009, 1-14. \\ B. Feichtinger, Ad maiorem gloriam Romae. Ideologie und Fiktion in der Historiographie des \\ Livius, Latomus 51, 1992, 3-33.
}

43 Vgl. OLD s.v. emo (3).

44 Vgl. dagegen Krafft (1998) 131. - Krafft versucht in anderem Zusammenhang nachzuweisen, dass die Postskripta generell keine Auswirkungen auf das Hauptnarrativ haben, sondern lediglich „im Anhang die bei der Vorentscheidung für die Erzählung ausgeschiedenen Versionen“ (133) aufzählen (131-33, mit zahlreichen Belegstellen). Ob dies haltbar ist, müsste für jede Textstelle einzeln untersucht werden. Für die behandelte Passage jedenfalls ist dies aus den genannten Gründen sicher nicht anzunehmen.

45 So auch die Interpretation Stevensons. - Vgl. Stevenson (2011) 179.

46 Ich verstehe den Begriff der ,if not-situation', wie er von Pausch (2011), 200-202 für die ,Beinahe-Episode“ verwendet wird. Pausch weist hier darauf hin, dass in Ab urbe condita auch sonst häufig solche if not-situations produziert werden - oft mithilfe eines Irrealis der Vergangenheit -, und führt zur Begriffsklärung eine Definition aus Grethlein (2006) an, die auch in diesem Kontext passend ist: „Dadurch, daß den Rezipienten der Verlauf der Handlung bekannt ist, entsteht leicht der Eindruck, die Handlung sei vorherbestimmt. Indem ,Beinahe-Episoden` einen alternativen Handlungsverlauf vorstellen, aber zur Tradition zurückkehren, machen sie es möglich, daß die Tradition gewahrt bleibt, aber trotzdem die Offenheit der Zukunft auf der Ebene der Handlung deutlich wird“ (Grethlein 2006, 280). So verstanden kann die Tarpeia-Episode bei Livius als eine - wenn auch implizite - Form kontrafaktischer Geschichtsschreibung gedeutet werden. 
G. Forsythe, A Critical History of Early Rome. From Prehistory to the First Punic War, Berkeley 2005.

H. Glareanus, In T. Livii Patavini decadas annotationes, Basel 1540 (online verfügbar: http:// www.erara.ch/bau_1/content/titleinfo/184115, letzter Zugriff: 17.06.2013).

J. B. Greenough (Hg.), Livy. Books I. and II., New Rochelle 1976 (=1891).

J. Grethlein, Das Geschichtsbild der Ilias. Eine Untersuchung aus phänomenologischer und narratologischer Perspektive (= Hypomnemata 163), Göttingen 2006.

J. Heurgon (Hg.), T. Livi Ab urbe condita liber primus, Paris 1963.

H. J. Hillen (Hg.), Livius, Römische Geschichte, Buch I-III, München 1987; Buch IV-VI, München 1991.

A. Keaveney, Livy and the Theatre. Reflections on the theory of Peter Wiseman, Klio 88, 2006, 510-515.

A. Klotz, Livius (9), RE XIII 1, 1972, 816-852.

B. Kowalewski, Frauengestalten im Geschichtswerk des T. Livius (= Beiträge zur Altertumswissenschaft 170), München u.a. 2002.

P. Krafft, Livius 4, 20 und Cossus' Konsulat, WS 111, 1998, 119-143.

D. S. Levene, Religion in Livy (= Mnemosyne Suppl. 127), Leiden u.a 1993.

G. B. Miles, Reconstructing early Rome, Ithaca 1995.

J. Moles, Livy's preface, in: J. D. Chaplin/C.S. Kraus (Hgg.), Livy (Oxford Readings in Classical Studies), Oxford 2009, 49-87 (= Nachdruck aus PCPhS 39, 1993, 141-68).

R. M. Ogilvie, A Commentary on Livy. Books 1-5, Oxford 1965.

- (Hg.), Titi Livi ab urbe condita libri 1-5, Oxford 1974.

D. Pausch, Der aitiologische Romulus. Historisches Interesse und literarische Form in Livius' Darstellung der Königszeit, Hermes 136, 2008, 38-60.

-, Livius und der Leser. Narrative Strukturen in ab urbe condita (= Zetemata 140), München 2011.

J. Poucet, Les préoccupations étiologiques dans la tradition «historique» sur les origins et les rois de Rome, Latomus 51, 1992, 281-314.

L. Richardson, A new topographical dictionary of ancient Rome, Baltimore u.a. 1992.

R. I. Ross, Livy. Book V, Bristol 1997.

R. B. Steele, The historical attitude of Livy, AJPh 25, 1904, 15-44.

T. Stevenson, Women of Early Rome as Exempla in Livy, Ab urbe condita, Book 1, CW 104, 2011, 175-189.

P. G. Walsh, Livy's preface and the distortion of history, AJPh 76, 1955, 369-83.

W. Weissenborn/H. J. Müller, Titi Livi ab urbe condita libri. Liber I, Berlin u. a. ${ }^{12} 1965$ ( $\left.={ }^{9} 1908\right)$; libri IV-V, Berlin u. a. ${ }^{8} 1965$ (= $\left.{ }^{6} 1896\right)$.

T. P. Wiseman, Roman Drama and Roman History, Exeter 1998.

S. Zimmermann, Tarpeia, DNP 12/1, 2002, 29. 